\title{
Transfer of a Gonococcal $\beta$-Lactamase Plasmid to Conjugation-deficient Neisseria cinerea Strains by Transformation
}

\author{
By CAROLINE ATTARDO GENCO $\dagger$ AND VIRGINIA L. CLARK* \\ Department of Microbiology and Immunology, School of Medicine and Dentistry, \\ University of Rochester, Rochester, NY 14642, USA
}

(Received 12 April 1988; revised 10 August 1988)

\begin{abstract}
We have previously shown that some strains of Neisseria cinerea can serve as recipients in conjugation $\left(\mathrm{Con}^{+}\right)$with Neisseria gonorrhoeae while others cannot (Con $\left.{ }^{-}\right)$. To determine if a replication defect contributes to the inability of certain strains of $N$. cinerea to serve as recipients in conjugation, we attempted to introduce a naturally occurring gonococcal $\beta$-lactamase plasmid into $N$. cinerea by transformation. Various methods were employed, and all proved unsuccessful. Since specific sequences are required for DNA uptake in transformation of $N$. gonorrhoeae, we constructed a number of hybrid plasmids containing $N$. cinerea chromosomal DNA inserted into the $N$. gonorrhoeae/Escherichia coli $\beta$-lactamase shuttle vector, pLES2. When nine randomly selected plasmids with inserts were used to transform an $N$. cinerea strain which did not accept the gonococcal $\beta$-lactamase plasmid by conjugation, transformants were observed with four of the hybrid plasmids. The presence of one of the hybrid plasmids, pCAG9, in transformants was confirmed by agarose gel electrophoresis, Southern hybridization, and $\beta$-lactamase production. When an $N$. gonorrhoeae donor strain containing pCAG9 was used in conjugation with several $N$. cinerea strains, only those strains that were previously shown to act as recipients could accept and maintain pCAG9. The ability of pCAG9 and the other three hybrid plasmids to transform $C^{-}$strains demonstrates that the $\beta$-lactamase plasmid can replicate in $\mathrm{Con}^{-}$strains, and, therefore, the $\mathrm{Con}^{-}$phenotype is due to a block in some other stage of the conjugation process.
\end{abstract}

\section{INTRODUCTION}

Since their discovery in 1976, penicillinase-producing Neisseria gonorrhoeae (PPNG) have been isolated at increasing frequencies around the world (Jaffe et al., 1981; Morgan, 1986). One of the potential modes for the spread of PPNG is conjugal transfer of a $\beta$-lactamase plasmid (Handsfield et al., 1982). The failure to contain the spread of PPNG strains raises the possibility that a non-pathogenic indigenous bacterial species could serve as an alternative reservoir for these plasmids. We have reported previously that some, but not all, strains of commensal Neisseria species can serve as recipients for the $\beta$-lactamase plasmid by conjugation with $N$. gonorrhoeae (Genco et al., 1984). What we observed was not simply a change in the magnitude of the conjugation frequency but rather an 'all or nothing' phenomenon. These results prompted further studies designed to define the nature of the inability of certain Neisseria strains to serve as recipients in conjugation with $N$. gonorrhoeae.

From studies with other conjugative plasmids (Broda, 1979) it has been shown that the process of conjugation can be divided into three events: (1) the collision event, ultimately resulting in contact formation between the donor and recipient cell, (2) transfer of plasmid DNA from the donor to the recipient cell, and (3) replication and processing of DNA in the recipient cell. Although little is known about the molecular events of the gonococcal conjugation system, we

† Present address: Sexually Transmitted Diseases Laboratory Program, Centers for Disease Control, Atlanta, GA 30333, USA. 
assume that conjugation into Neisseria cinerea could be blocked at any of these stages. The replication of the transferred plasmid in the new host is essential, since if it cannot replicate it will be lost during successive cell divisions. The present investigation was undertaken to determine if a replication defect contributes to the inability of some strains of $N$. cinerea to serve as recipients in conjugation with $N$. gonorrhoeae for the $\beta$-lactamase plasmid. Experiments were therefore performed to attempt to introduce gonococcal $\beta$-lactamase plasmids into $N$. cinerea conjugation deficient $\left(\mathrm{Con}^{-}\right)$strains by transformation. The successful maintenance of gonococcal $\beta$-lactamase plasmids in $\mathrm{Con}^{-}$strains indicates that these plasmids can replicate in these strains.

\section{METHODS}

Bacterial strains and plasmids. These are listed in Tables 1 and 2. Neisseria strains were maintained on a daily basis by streaking colonies on GC medium base (Difco) containing Kellogg's supplement (GCK) (White \& Kellogg, 1965). Large volumes were grown in GCK broth containing $\mathrm{NaHCO}_{3}\left(420 \mathrm{mg} \mathrm{1}^{-1}\right)$ at $37^{\circ} \mathrm{C}$ with aeration. Escherichia coli was grown on MacConkey agar at $37^{\circ} \mathrm{C}$ or in liquid culture (LB broth) at $37^{\circ} \mathrm{C}$ with aeration.

Neisseria and $E$. coli strains were screened for their plasmid content by a rapid isolation method (Davis $e t$ al., 1980), and plasmids were visualized by staining with ethidium bromide.

Plasmid purification. Plasmid DNA was isolated by ethidium bromide/CsCl isopycnic density gradient centrifugation by the method of Guerry et al. (1973), as modified by Stein et al. (1983b).

Conjugation and transformation. Conjugations were performed by the filter mating technique as previously described (Genco et al., 1984). N. cinerea and $N$. gonorrhoeae strains were transformed with plasmid DNA by a procedure originally designed for $N$. gonorrhoeae (Sox et al., 1979) and transformants were selected on GCK agar containing $0.25 \mu \mathrm{g}$ penicillin $\mathrm{G} \mathrm{ml}^{-1}$. Transformants and transconjugants were confirmed by detection of $\beta$ -

Table 1. Bacterial strains used in this study

\begin{tabular}{|c|c|c|c|}
\hline Organism & Strain & $\begin{array}{c}\text { Relevant } \\
\text { phenotype* }\end{array}$ & Source \\
\hline N. gonorrhoeae & $\begin{array}{l}\text { CDC67 } \\
\text { CDC66 } \\
\text { RUN4062 }\end{array}$ & $\begin{array}{l}\text { Tra }^{+} \text {Bla }^{+} \\
\text {Tra }^{+} \\
\text {Tra }^{+} \text {Bla }^{+}\end{array}$ & $\begin{array}{l}\text { CDC, Atlanta } \\
\text { CDC, Atlanta } \\
\text { Transformation of pCAG9 into CDC66 }\end{array}$ \\
\hline N. cinerea & $\begin{array}{l}\text { RUN4029 } \\
\text { RUN4030 } \\
\text { RUN4031 } \\
\text { RUN4032 } \\
\text { RUN4033 }\end{array}$ & $\begin{array}{l}\text { Bla- Con }^{-} \\
\text {Bla- Con }^{+} \\
\text {Bla- Con }^{+} \\
\text {Bla- Conn }^{-} \\
\text {Bla- Con }^{+}\end{array}$ & $\begin{array}{l}\text { NRL } 30003 \\
\text { NRL } 32165 \\
\text { NRL } 32824 \\
\text { NRL } 33295 \\
\text { NRL } 32828\end{array}$ \\
\hline E. coli & JM83 & $\mathrm{Lac}^{-}$ & J. Messing \\
\hline
\end{tabular}

* $\mathrm{Tra}^{+}$strains contain the gonococcal conjugal plasmid, pLE2451. Bla ${ }^{+}$indicates that the strain produces a $\beta$ lactamase and contains either pFT1 (CDC67) or pCAG9 (CDC66). Con ${ }^{-}$and Con $^{+}$indicate the inability or capability, respectively, of the strain to receive the $\beta$-lactamase plasmid by conjugation with $N$. gonorrhoeae. Lac ${ }^{-}$ indicates inability to utilize lactose as an energy source.

+ NRL denotes Neisseria Reference Laboratory.

Table 2. Plasmids used in this study

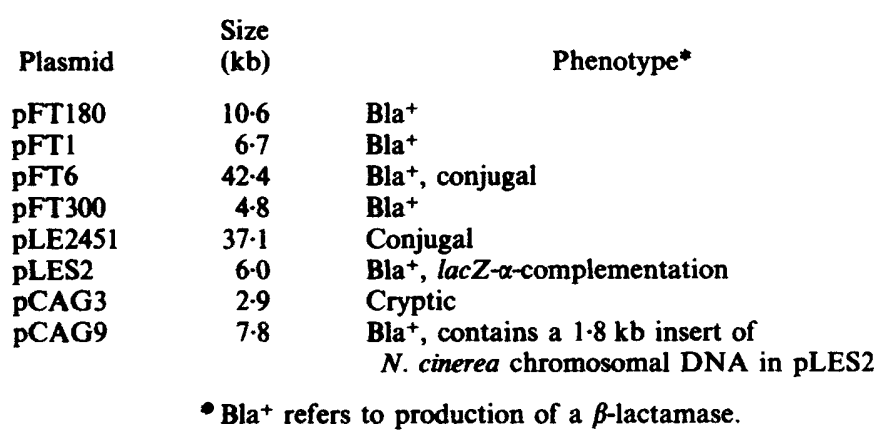


lactamase activity with the chromogenic cephalosporin nitrocefin (O'Callaghan et al., 1972). E. coli was transformed using a $\mathrm{CaCl}_{2}$ procedure (Cohen et al., 1972).

Construction of hybrid plasmids. This was done by inserting $N$. cinerea chromosomal DNA into the lac $Z-\alpha-$ complementation region of the $N$. gonorrhoeae/E. coli shuttle vector pLES2 (Stein et al., 1983a). Ligation mixtures were used to transform $E$. coll JM83 with selection for transformants on MacConkey agar containing $30 \mu \mathrm{g}$ ampicillin $\mathrm{ml}^{-1}$.

Southern hybridization. DNA was transferred from agarose gets to nitrocellulose as described by Wahl et al. (1979). Probe DNA was labelled with ${ }^{32}$ P-dCTP by niek-translation (Maniatis et al., 1982), and hybridization was performed at $65^{\circ} \mathrm{C}$ for $16 \mathrm{~h}$ as described by Southern (1975).

\section{RESULTS}

\section{Transformation of $N$. cinerea with gonococcal $\beta$-lactamase plasmid DNA}

We used $N$. cinerea in these studies of $\beta$-lactamase plasmid replication in $\mathrm{Con}^{-}$strains because the $\beta$-lactamase plasmids are transferred at high frequency by conjugation and stably maintained in $N$. cinerea $\mathrm{Con}^{+}$strains (Genco et al., 1984). Several different gonococcal $\beta$ lactamase plasmids (pFT1, pFT180, pLES2 and pFT6) were used in attempts to transform $N$. cinerea $\mathrm{Con}^{-}$strains, by a number of different methods, all of which proved unsuccessful. These included $\mathrm{CaCl}_{2}$ transformation (Cohen et al., 1972), $\mathrm{RbCl}_{2}$ transformation (Hanahan, 1983), a transformation procedure originally described for Haemophilus influenzae (Stuy, 1979), and a standard procedure used for $N$. gonorrhoeae (Sox et al., 1979).

Plasmid transformation is relatively inefficient in $N$. gonorrhoeae (Cannon \& Sparling, 1984), and specific sequences are required for DNA uptake (Graves et al., 1982). If specific uptake sequences are also required in $N$. cinerea, supplying these sequences should overcome the inability of the $\beta$-lactamase plasmid to transform this species. Hybrid plasmids (pCAG2, pCAG4, and pCAG6-12) were constructed containing $N$. cinerea chromosomal DNA inserted into the BamHI site of the $N$. gonorrhoeae/E. coli $\beta$-lactamase shuttle vector, pLES2 (Stein et al., $1983 a$ ). pLES2 contains the lacZ- $\alpha$-complementation region from pUC9 that allows the direct visualization of plasmids containing inserts in the BamHI site after transformation into the appropriate $E$. coli host (JM83), by the Lac ${ }^{-}$phenotype. $N$. cinerea RUN4030 chromosomal DNA was inserted into pLES2, the ligated mixture was used to transform $E$. coli JM83, and Amp ${ }^{r}$ transformants were screened for the Lac $^{-}$phenotype. Approximately $75 \%$ of the Amp ${ }^{r}$ transformants were found to be $\mathrm{Lac}^{-}$.

The presence of $N$. cinerea DNA in one of the hybrid plasmids, pCAG9, was confirmed by a dot-blot procedure. pCAG9 hybridized to pCAG9, pLES2, and to DNA from two strains of $N$. cinerea and three strains of $N$. gonorrhoeae; some hybridization to Neisseria flava and Branhamella catarrhalis was also observed (data not shown). The pLES2 vector hybridized only to pCAG9, pLES2 and pCAG2, and not to the chromosomal DNA from these Neisseria spp. (data not shown).

When nine different hybrid plasmids containing $N$. cinerea DNA inserts (ranging from $0.2 \mathrm{~kb}$ to $1.9 \mathrm{~kb}$ ) were used to transform $\mathrm{Con}^{-} N$. cinerea RUN4029, transformants were observed with four of the plasmids, pCAG4, pCAG9, pCAG11 and pCAG12 (data not shown). The transformation frequencies varied somewhat for the four plasmids $\left(1.5 \times 10^{-5}\right.$ to $\left.7.0 \times 10^{-7}\right)$, and did not correlate with the size of the DNA insert. All transformants produced significant levels of $\beta$-lactamase activity as measured by nitrocefin cleavage.

The presence of pCAG9 in the transformants was confirmed by agarose gel electrophoresis (Fig. $1 a$ ) and by Southern hybridization (Fig. 1b). The agarose gel was bi-directionally blotted onto nitrocellulose to generate two identical blots, one of which was hybridized with ${ }^{32} \mathrm{P}$-labelled pCAG9 (Fig. $1 b$ ) and the other with ${ }^{32}$ P-labelled pCAG3 (data not shown). pCAG3 is the resident plasmid of the host strain, $N$. cinerea RUN4029. pCAG9 hybridized to itself, to its parent plasmid pLES2, and to plasmid DNA in the $N$. cinerea RUN4029 transformants. Hybridization to chromosomal DNA from $N$. cinerea RUN4029 was not evident; significant hybridization would not be observed because of low concentrations of chromosomal DNA in the gel. In the blot probed with pCAG3, pCAG3 hybridized to plasmid DNA in the host strain and all $N$. cinerea RUN4029 transformants, at the size of pCAG3 but not of pCAG9 (data not 

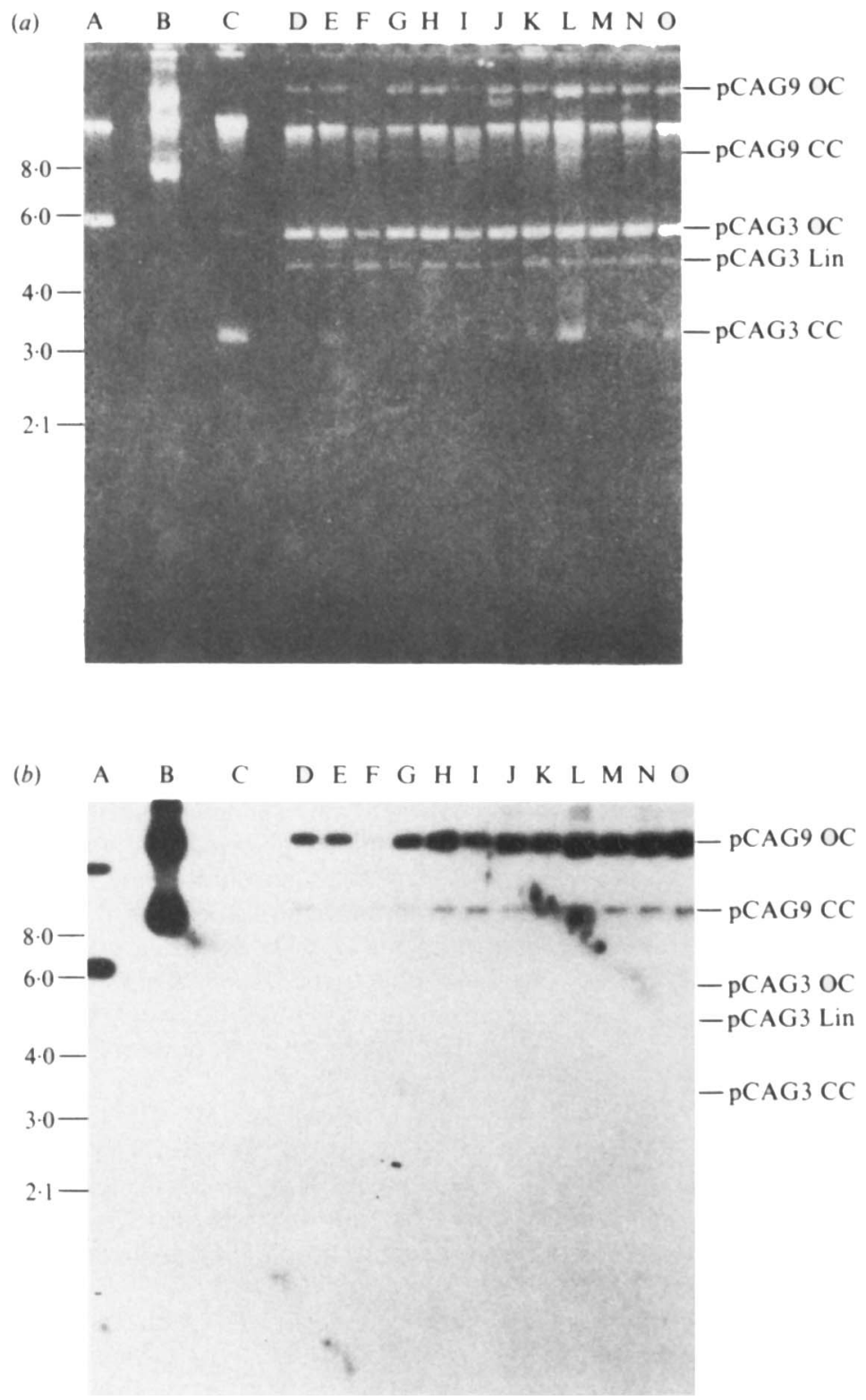

Fig. 1. Presence of pCAG9 in $N$. cinerea RUN4029 transformants. Plasmid DNA was isolated, as described in Methods, by $\mathrm{CsCl}$ density gradient centrifugation for plasmids pLES2 (lane A) and pCAG9 (lane B) or by plasmid screens for $N$. cinerea RUN4029 (lane C) and 12 transformants of RUN4029 containing pCAG9 (lanes D-O). The DNA was separated on a $1 \%(w / v)$ agarose gel, stained with ethidium bromide $(a)$, bidirectionally blotted onto nitrocellulose and hybridized with ${ }^{32}$ P-labelled pCAG9 $(b)$ or ${ }^{32}$ P-labelled pCAG3 (data not shown). The sizes (kb) of supercoiled plasmid DNA markers (Bethesda Research Laboratories) are shown on the left. The positions of open circular (OC) and closed circular $(\mathrm{CC})$ forms of pCAG3 and pCAG9 prepared by the plasmid screening method are indicated on the right (Lin, linear). The mobility of pCAG9 prepared by the $\mathrm{CsCl}$ method is somewhat faster than what is obtained by the plasmid screening method.

shown). The presence of this plasmid confirmed the identify of the $N$. cinerea strain. Hybridization of pCAG3 to pLES2 or pCAG9 was not observed. The band in Fig. 1(a), lane J, running just below the pCAG9 OC, did not hybridize with either pCAG9 or pCAG3. 
Table 3. Transformation and conjugation frequencies for $p C A G 9$

\begin{tabular}{lccc}
\multicolumn{1}{c}{ Donor* } & Recipient $\dagger$ & $\begin{array}{c}\text { Transformation } \\
\text { frequency }\end{array}$ & $\begin{array}{c}\text { Conjugation } \\
\text { frequency }\end{array}$ \\
E. coli JM83(pCAG9) & RUN4029 & $4.0 \times 10^{-6}$ & ND \\
$N$. cinerea RUN4029(pCAG9) & RUN4029 & $4.2 \times 10^{-5}$ & ND \\
$N$. gonorrhoeae RUN4062(pCAG9) & RUN4029 & $1.0 \times 10^{-6}$ & $<10^{-9}$ \\
& RUN4030 & $<10^{-8}$ & $7.3 \times 10^{-5}$ \\
& RUN4031 & $<10^{-8}$ & $1.1 \times 10^{-5}$
\end{tabular}

ND, Not done. E. coli JM83(pCAG9) and N. cinerea RUN4029(pCAG9) were not used as donors in conjugation because they do not contain a conjugal plasmid.

- The strain from which the plasmid was isolated for transformation experiments, or the strain which contained the conjugal plasmid as well and served as a donor in conjugation.

† The $N$. cinerea strain into which pCAG9 was introduced by transformation, or the strain which interacted with a donor strain in conjugation.

† Transformation frequency and conjugation frequency are expressed as transformants and transconjugants, respectively, per input cell and are representative of three separate experiments.

\section{Confirmation of unique inserts}

Since four hybrid plasmids containing $N$. cinerea DNA inserts of different size were able to transform $\mathrm{Con}^{-} N$. cinerea RUN4029, it is unlikely that this was due to the cloning of a replication origin from RUN4029. To confirm that these four inserts were unique, we hybridized pCAG9 to Sau3A digests of the four plasmids. pCAG9 hybridized strongly to all undigested plasmids, to vector fragments and to a band of $1.8 \mathrm{~kb}$ in the Sau3A-digested pCAG9. No hybridization of pCAG9 to the inserts of the three other hybrid plasmids was detected. The ability of pCAG9 and the three other hybrid plasmids, but not pLES2, to transform $N$. cinerea RUN4029 Con- cells is therefore most likely not due to the insertion of sequences mediating replication of pLES2 in $N$. cinerea, but rather to the insertion of sequences required for DNA uptake by transformation.

\section{Transfer of $P C A G 9$ into $N$. cinerea recipients by transformation and conjugation}

The experiments described above demonstrated that pCAG9 could be introduced into $N$. cinerea RUN4029 Con $^{-}$cells by transformation. To determine if pCAG 9 could be introduced into $N$. cinerea $\mathrm{Con}^{-}$strains by conjugation, the plasmid was used to transform $N$. gonorrhoeae CDC66, creating a donor, RUN4062, which could be used in conjugation with $N$. cinerea recipients. The Con- $N$. cinerea strain RUN4029 was not able to accept pCAG9 by conjugation from the $N$. gonorrhoeae RUN4062 donor (Table 3). $N$. cinerea RUN4030 and RUN4031 (Con ${ }^{+}$ strains) were both able to serve as recipients in transfer of the hybrid plasmid, pCAG9, from $N$. gonorrhoeae RUN4062 by conjugation. The frequency of conjugal transfer of pCAG9 into these strains was comparable to the frequency of transfer of pFT1 (Genco et al., 1984). pCAG9 DNA isolated from $E$. coli JM83, $N$. cinerea RUN4029 or $N$. gonorrhoeae RUN4062 could transform $N$. cinerea $\mathrm{Con}^{-}$recipients (Table 3). The inability of pCAG9 DNA isolated from $N$. gonorrhoeae RUN4062 to transform either $N$. cinerea RUN4030 or RUN4031 may be due to restriction of plasmid DNA during transformation or to a low competence level, analogous to that of type 4 gonococci, in these strains.

\section{DISCUSSION}

The transformation of $N$. cinerea RUN4029 by gonococcal $\beta$-lactamase plasmid DNA indicated that this plasmid can be replicated in a Con- strain. That pCAG9 was present as a plasmid, and not integrated into the chromosome, was evident from agarose gels and Southern hybridizations. The failure to transfer the $\beta$-lactamase plasmid into $N$. cinerea RUN4029 by conjugation was not due to lack of expression of the gonococcal $\beta$-lactamase gene, as seen by measurements of $\beta$-lactamase activity in the transformants. 
In other systems, the ability of a transferred plasmid to replicate in diverse bacteria appears to be the most important factor determining host range (Guiney, 1982). Conjugative plasmids differ in their ability to transfer and replicate in unrelated bacteria. Plasmids of the incompatibility group P (IncP) have a very broad host range and can be transferred to most Gram-negative organisms (Chikami et al., 1985). These plasmids must possess a conjugation system that facilitates transfer between unrelated bacteria, together with a replication system that maintains the plasmid in each host. In contrast, plasmids of the IncFI and IncFII groups have a narrower host range, in that they can be transferred among the Enterobacteriaceae but not to Pseudomonas aeruginosa (Datta \& Hedges, 1972). The basis for the host range limitation appears to be an inability of these plasmids to replicate in $P$. aeruginosa (Guiney, 1982).

The ability of pCAG9 to replicate in $N$. cinerea RUN4029 does not appear to be due to the unlikely event of cloning of an origin for replication. Of nine randomly selected hybrid plasmids, four could transform RUN4029. This frequency would be too high to represent cloned replication origins, unless they all represented progeny of the same clone. This was not the case, as pCAG9 did not share homology in its inserted sequence with the other three hybrid plasmids. Even if a replication origin had been cloned, it would not alter our conclusion that replication is not the stage at which conjugation is blocked, since pCAG9 still cannot be transferred to $N$. cinerea RUN4029 by conjugation.

The fact that pCAG9 isolated in an $N$. gonorrhoeae host could be transformed into $N$. cinerea RUN4029 Con- organisms suggests that the inability to transfer the plasmid from this donor to this strain by conjugation is not due to restriction of gonococcal $\beta$-lactamase plasmid DNA. Some restriction of pCAG9 may occur during transformation; however, it is not great enough to explain the complete inhibition of conjugal transfer. Stein et al. (1988) have recently demonstrated that host-mediated restriction can prevent the gonococcus from acquiring DNA via transformation but not via conjugation.

In summary, the ability of $N$. cinerea RUN4029 to accept pCAG9 by transformation, but not by conjugation, suggests that the barrier to conjugation in $N$. cinerea RUN4029 is not the inability to replicate the gonococcal $\beta$-lactamase plasmid. The $\mathrm{Con}^{-}$phenotype of the $N$. cinerea strains is also probably not due to restriction of plasmid DNA after transfer. Therefore, these Con $^{-}$strains must be deficient in another, probably earlier, stage in the conjugation process. Results described in the accompanying paper (Genco \& Clark, 1988) suggest that these $\mathrm{Con}^{-} N$. cinerea strains may lack a component in the outer membrane that is required for cells to serve as recipients in conjugation with $N$. gonorrhoeae.

This study was supported by US Public Health Service grant AI-11709 from the National Institute of Allergy and Infectious Diseases.

\section{REFERENCES}

Broda, P. (1979). Conjugal transfer. In Plasmids, pp. 83-99. San Francisco: W. H. Freeman.

Cannon, J. G. \& Sparlnug, P. F. (1984). The genetics of the gonococcus. Annual Review of Microbiology 38, 111-133.

Chiram, G. K., Guiney, D. G., Schmidhuser, T. J. \& Helinski, D. R. (1985). Comparison of $10 \mathrm{IncP}$ plasmids: homology in the regions involved in plasmid replication. Journal of Bacteriology 162, 656660.

Cohen, S. N., Chang, A. C. Y. \& Hsu, L. (1972). Nonchromosomal antibiotic resistance in bacteria: genetic transformation of Escherichia coli by $\mathbf{R}$ factor DNA. Proceedings of the National Academy of Sciences of the United States of America 69, 2110 2114.

DATTA, N. \& Hedges, R. W. (1972). Host range of R factors. Journal of General Microbiology 70, 453-460.
DAvis, R. W., Botsten, D. \& Roth, J. R. (1980). A Mamual for Genetic Engineering: Advanced Bacterial Genetics, pp. 120-123. Cold Spring Harbor, NY: Cold Spring Harbor Laboratory.

GenCo, C. A. \& ClaRK, V. L. (1988). Role of outermembrane proteins and lipopolysaccharide in conjugation between Neisseria gonorrhoeae and Neisseria cinerea. Journal of General Microbiology 134, 32853294.

Genco, C. A., KnapP, J. S. \& Clark, V. L. (1984). Conjugation of plasmids of Neisseria gonorrhoeae to other Neisseria species: potential reservoirs for the beta-lactamase plasmid. Journal of Infectious Diseases 150, 397-401.

Graves, J. F., Biswas, G. D. \& SParling, P. F. (1982). Sequence-specific DNA uptake in transformation of Neisseria gonorrhoeae. Journal of Bacteriology 152, 1071-1077. 
Guerry, P., Leblanc, D. J. \& Falkow, S. (1973). General method for the isolation of plasmid deoxyribonucleic acid. Journal of Bacteriology 116, 1064 1066.

GuINEY, D. G. (1982). Host range of conjugation and replication functions of the Escherichia coli sex plasmid FLac: comparison with the broad hostrange plasmid RK2. Journal of Molecular Biology 162, 699-703.

Hanahan, D. (1983). Studies on transformation of Escherichia coli with plasmids. Journal of Molecular Biology 166, 557-580.

HANDSField, H. H., SANDSTrom, E. G., KNAPP, J. S., Perine, P. L., Whittington, W. L., SAYers, D. E. \& HoLmes, K. K. (1982). Epidemiology of penicillinase-producing Neisseria gonorrhoeae infections. Analysis by auxotyping and serotyping. New England Journal of Medicine 306, 950-954.

JAFFE, H. W., BIDDle, J. W., Johnson, S. R. \& WIESNER, P. J. (1981). Infections due to penicillinase-producing Neisseria gonorrhoeae in the United States: 1976-1980. Journal of Infectious Diseases 144, 191-197.

Maniatis, T., Fritsch, E. F. \& Sambrook, J. (1982). Molecular Cloning, a Laboratory Manual. Cold Spring Harbor, NY: Cold Spring Harbor Laboratory.

MorgaN, R. (1986). PPNG - United States, Florida. Journal of the American Medical Association 225, 1674-1679.

O'Callaghan, C. H., Morris, C. H., Kirby, S. M. \& SHINGLeR, A. H. (1972). A novel method for detection of beta-lactamase by using a chromogenic cephalosporin substrate. Antimicrobial Agents and Chemotherapy 1, 283-288.
SOUTHERn, E. M. (1975). Detection of specific sequences among fragments separated by gel electroporesis. Journal of Molecular Biology 90, 503-517.

SoX, T. E., Mohammed, W. \& SParling, P. F. (1979). Transformation-derived Neisseria gonorrhoeae plasmids with altered structure and functions. Journal of Bacteriology 138, 510-516.

Stein, D. C., Silver, L. E., Clark, V. L. \& Young, F. E. (1983a). Construction and characterization of a new shuttle vector, pLES2, capable of functioning in Escherichia coli and Neisseria gonorrhoeae. Gene 25, 350-354.

Sten, D. C., Young, F. E., Tenover, F. C. \& Clark, V. L. $(1983 b)$. Characterization of a chimeric betalactamase plasmid of Neisseria gonorrhoeae which can function in Escherichia coli. Molecular and General Genetics 189, 77-84.

StenN, D. C., Gregorre, S. \& Piekarowicz, A. (1988). Restriction of plasmid DNA during transformation but not conjugation in Neisseria gonorrhoeae. Infection and Immunity 56, 112-116.

STUY, G. H. (1979). Plasmid transfer in Haemophilus influenzae. Journal of Bacteriology 139, 520-529.

WAHL, G. F., Stern, M. \& Stark, G. R. (1979). Efficient transfer of large DNA fragments from agarose gels to diazobenzyloxymethyl-paper and rapid hybridization by using dextran sulfate. Proceedings of the National Academy of Sciences of the United States of America 76, 3683-3687.

White, L. A. \& KellogG, D. S. (1965). Neisseria gonorrhoeae identification in direct smears by a fluorescent antibody-counterstain method. Applied Microbiology 13, 171-174. 